\title{
Multiple changes in gene expression in chronic human Achilles tendinopathy
}

\author{
Deborah Ireland ${ }^{\mathrm{a}, *}$, Rebecca Harrall ${ }^{\mathrm{a}}$, Valerie Curry ${ }^{\mathrm{a}}$, Graham Holloway ${ }^{\mathrm{b}}$, \\ Roger Hackney ${ }^{\mathrm{c}}$, Brian Hazleman ${ }^{\mathrm{a}}$, Graham Riley ${ }^{\mathrm{a}}$ \\ ${ }^{a}$ Rheumatology Research Unit, Box 194, E6, Addenbrooke's Hospital, Hills Road, Cambridge CB2 2QQ, UK \\ ${ }^{\mathrm{b}}$ Back on Track Sports Injury Clinic, The Ridgeway Hospital, Moormead Road, Wroughton, Swindon, UK \\ ${ }^{\mathrm{c}}$ Leeds General Infirmary, Great George Street, Leeds, UK
}

Received 27 April 2000; received in revised form 25 January 2001; accepted 2 February 2001

\begin{abstract}
Atlas $^{\mathrm{TM}}$ cDNA cell interaction arrays $(\mathrm{CLONTECH})$ were used to examine degenerate tissue from four patients with Achilles tendon disorders, in order to identify changes in expression of genes important in cell-cell and cell-matrix interactions. The greatest difference between normal (post-mortem) and degenerate tissue samples was in the level of MMP-3 (stromelysin) mRNA, which was down-regulated in all the degenerate samples. Quantitative RT-PCR assay of RNA extracted from paired 'normal' and degenerate Achilles tendon tissue samples taken from tendons during surgery mirrored the results of the arrays. Levels of MMP-3 mRNA were lower, whereas levels of type-I and type-III collagen mRNAs were significantly higher, in the degenerate compared to the 'normal' samples. Immunoblotting of proteins extracted from the same tendon samples showed that three of four normal tissue samples taken from individuals without apparent tendon disorder had much higher levels of MMP-3 protein than 'normal' or degenerate samples from patients with tendinosis. We suggest that MMP-3 may play an important role in the regulation of tendon extracellular matrix degradation and tissue remodelling. (c) 2001 Elsevier Science B.V./International Society of Matrix Biology. All rights reserved.
\end{abstract}

Keywords: Gene expression; mRNA; Tendinopathy

\section{Introduction}

The most common finding during surgery for chronic Achilles tendon pain is intratendinous degeneration or tendinosis (Astrom and Rausing, 1995). Degenerate areas differ macroscopically from the surrounding tissue by their soft, grey or yellowish, nonglistening appearance (Dahmen, 1964). In a study of 342 operations for chronic Achilles tendon pain, $72 \%$

\footnotetext{
*Corresponding author. Tel.: +44-1223-216050; fax: +44-1223217838.

E-mail address: dci20@mole.bio.cam.ac.uk (D. Ireland).
}

of the tendons were found to have areas of intratendinous degeneration while the rest showed no macroscopic pathology (Astrom, 1998).

Tendinosis is also found in the absence of symptoms. One-third of people over 35 years of age, with no history of tendon problems, have degenerative tendon changes (Kannus and Jozsa, 1991). Also, many patients with ruptured Achilles tendons have no symptoms beforehand, but degenerate tissue surrounds the torn tendon tissue (Astrom and Rausing, 1995). The tendinosis is generally thought to cause the rupture not result from it (Kannus and Jozsa, 1991; Gibson, 1998).

Histopathological examination of normal Achilles 
tendon tissue samples taken at autopsy showed dense straight or slightly wavy parallel-packed collagen fibres with rows of cells, vessels and nerves located between the fibre bundles (Movin et al., 1997a). In contrast, degenerate samples taken from 40 patients with painful Achilles tendons showed: increased amounts of non-collagenous matrix; alterations in the structure and arrangement of collagen fibres; and focal variations in cellularity and vascularization. There was no infiltration of inflammatory cells in these late stage samples. Recognising these features of tendinosis, Khan et al. (1999) concluded that effective treatment of athletes with tendinopathies must target this non-inflammatory condition. However, the aetiology of tendinosis is unknown and the changes in degenerate tissues have not been studied extensively.

The architecture of a tissue depends on cellular interactions, both homo- and heterotypic cell-cell interactions and cell-matrix interactions (Petruzzelli et al., 1999). Tenocytes produce both the components of the extracellular matrix and also the enzymes that degrade them. They produce adhesion molecules that mediate cellular interactions and influence cell signalling, transcription and response to growth factors. Mis-expression of matrix or adhesion molecules is the underlying cause of many developmental or degenerative disorders (Lukashev and Werb, 1998; Petruzzelli et al., 1999).

Regulation of tissue phenotype involves changes in levels of individual mRNAs and the translation and survival of their cognate proteins. We have used cDNA arrays to examine the expression of a large number of genes involved in cell interactions in both degenerate and normal human Achilles tendon tissue samples in order to identify differences that could account for the degenerate phenotype. We have compared levels of mRNA and protein for matrix metalloproteinase MMP-3 in normal and degenerate samples.

\section{Results}

\subsection{RNA extraction and quantification}

The four samples of degenerate tendon tissue obtained from patients undergoing surgery for Achilles tendon disorders, and the three samples of normal control tissue taken from a human cadaver, were quite large and so three small pieces (each approx. 50 mg wet weight) from each sample were used for RNA extraction. The paired samples of degenerate and normal tissue taken from the same tendon were smaller. The average size of the first set of six paired samples used for RNA extraction was $14 \mathrm{mg}$ wet weight (range 9-22 mg). The average yield of total
RNA from the normal tissue samples was $45 \mathrm{ng} / \mathrm{mg}$ (range 30-57) and the average yield from the degenerate tissue samples was $51 \mathrm{ng} / \mathrm{mg}$ (range 25-76). Gel analysis of the RNA showed it to be largely undegraded.

\section{2. cDNA expression arrays}

${ }^{32}$ P-labelled cDNA probes with total counts per minute of $1.7-6.9 \times 10^{6}$ were obtained from 1 to $2 \mu \mathrm{g}$ total RNA using the Atlas ${ }^{\mathrm{TM}}$ array reagents. The strongest signals, including those for GAPDH, were visible on the film after overnight exposure of the arrays, and the remaining signals were visible after 1 week. No signal corresponding to genomic DNA was seen on any of the arrays.

The Atlas human cell-interaction arrays include 265 genes from different areas of cell interaction research. Fig. 1 shows examples of autoradiographs produced from normal and degenerate tendon tissue samples. In all, 17 genes were up-regulated and 23 down-regulated in all the degenerate tissue samples (greater than two-fold increase or decrease in normalised signal, or detection in only normal or degenerate tissue). Nine genes were up-regulated and two down-regulated only in R1 and R2, and one gene was up-regulated and two down-regulated only in $\mathrm{T} 1$ and T2. Normalisation of the signals for specific genes to those for beta-actin gave similar results to those obtained using GAPDH. Fig. 2 shows the genes for ECM and cell-adhesion molecules differentially expressed in degenerate and normal human Achilles tendon tissue samples and includes some genes that were differentially expressed in only three of the four degenerate tissue samples. The results for the collagens, non-collagen matrix components and matrix metalloproteinases (MMPs) and their inhibitors are shown in more detail in Tables 1, 2 and 3.

Table 1

Cell-matrix interactions - collagens

\begin{tabular}{llllll}
\hline Collagen types & \multicolumn{5}{l}{ Tendon sample } \\
& Normal & T1 & T2 & R1 & R2 \\
\hline I & 0.16 & $\mathbf{2 . 6}$ & $\mathbf{2 . 5}$ & $\mathbf{2 . 3}$ & $\mathbf{0 . 8 9}$ \\
II $\alpha-1$ & & & & & \\
III pro- $\alpha-1$ & 0.06 & $\mathbf{2 . 6}$ & $\mathbf{1 . 9}$ & $\mathbf{2 . 4}$ & $\mathbf{1 . 0 2}$ \\
IV $\alpha$ & & & & & \\
IV $\alpha-3$ & & & & & \\
IX $\alpha-1$ & & & & & \\
VI $\alpha-1$ & 0.31 & $\mathbf{1 . 5}$ & $\mathbf{1 . 2 6}$ & $\mathbf{1 . 0 9}$ & $\mathbf{0 . 7 6}$ \\
VI $\alpha-2$ & & & & & \\
VI $\alpha-3$ & 0.11 & $\mathbf{1 . 5}$ & $\mathbf{1 . 0}$ & $\mathbf{1 . 0 8}$ & $\mathbf{0 . 6 0}$ \\
VIII $\alpha-1$ & & & $\mathbf{0 . 1 3}$ & $\mathbf{0 . 1 9}$ & $\mathbf{0 . 1 2}$ \\
XI $\alpha-1$ & & & & & 0.10 \\
XI pro- $\alpha-2$ & 0.16 & & & & \\
XVI $\alpha-1$ & & $\mathbf{0 . 1 9}$ & $\mathbf{0 . 2 3}$ & $\mathbf{0 . 2 3}$ & $\mathbf{0 . 0 9}$ \\
XVIII $\alpha$ & & & & $\mathbf{0 . 0 9}$ & \\
\hline
\end{tabular}




\section{3. $Q C-R T-P C R$ and quantitative $R T-P C R$ using the GeneAmp 5700 sequence detection system}

QC-RT-PCR assays for type-I and type-III collagen mRNAs in six pairs of Achilles tendon samples mirrored the results of the arrays. All of the six tendons showed increased type-III collagen mRNA in the degenerate tissue samples and five of the six tendons showed increased type-I collagen mRNA in the degenerate tissue samples.

A comparison of GAPDH mRNA levels in the first six pairs of tendon samples obtained by using duplicate samples in QC-RT-PCR and real-time quantitative RT-PCR showed good concordance between the two sets of results. The coefficients of variation of the real-time assays ranged from 2.8 to $48 \%$. Clearly, more identical replicates were needed to give enough precision to distinguish two-fold differences in mRNA levels between paired tendon samples. Real-time RTPCR of RNAs from five more pairs of samples, using five identical replicates of a single RNA preparation per sample, showed significantly increased type-III collagen mRNA in each degenerate sample compared to its paired 'normal' sample when the data were analysed using the approximate test. Three of the pairs also had significantly increased type-I collagen mRNA in the degenerate samples. Since only one pair of samples could be taken from each patient, the results of the two methods of RT-PCR were pooled to give eleven pairs and the ratios degenerate/'normal'

Table 2

Cell-matrix interactions - other matrix components

\begin{tabular}{llllll}
\hline Matrix components & \multicolumn{5}{l}{ Tendon sample } \\
& Normal & $\mathrm{T} 1$ & $\mathrm{~T} 2$ & $\mathrm{R} 1$ & $\mathrm{R} 2$ \\
\hline Fibronectin & 0.05 & & & $\mathbf{0 . 1 3}$ & $\mathbf{0 . 1 1}$ \\
Laminin $\alpha 4$ & & $\mathbf{0 . 5 4}$ & $\mathbf{0 . 4 1}$ & $\mathbf{0 . 5 6}$ & $\mathbf{0 . 4 9}$ \\
LAMB2 & & 0.11 & 0.1 & 0.03 & \\
Laminin & & & & & \\
Laminin B1 & & & & $\mathbf{0 . 0 9}$ & $\mathbf{0 . 0 8}$ \\
Laminin B2 & 0.07 & 0.08 & & 0.08 & 0.09 \\
Laminin 37 kDa & 0.13 & 0.16 & & $\mathbf{0 . 3 1}$ & $\mathbf{0 . 2 8}$ \\
Netrin-2 & & & & & \\
Nidogen & & & & $\mathbf{0 . 1 4}$ & $\mathbf{0 . 0 6}$ \\
SPARC & 0.35 & $\mathbf{2 . 5}$ & $\mathbf{2 . 2 6}$ & $\mathbf{1 . 7 7}$ & $\mathbf{0 . 8 6}$ \\
Tenascin-C & 0.14 & $\mathbf{0 . 8 4}$ & $\mathbf{0 . 6 8}$ & $\mathbf{0 . 7}$ & $\mathbf{0 . 5 5}$ \\
Tenascin-R & & & & & \\
Thrombospondin 1 & & & & & \\
Thrombospondin 2 & 0.05 & & $\mathbf{0 . 1 2}$ & $\mathbf{0 . 2 1}$ & 0.06 \\
Versican & 0.33 & $\mathbf{1 . 3 2}$ & $\mathbf{0 . 7 3}$ & $\mathbf{0 . 8 5}$ & 0.44 \\
Vitronectin & & & & & \\
HSPG2 & 0.12 & $\mathbf{0 . 4 5}$ & $\mathbf{0 . 3 9}$ & $\mathbf{0 . 4 2}$ & $\mathbf{0 . 3 6}$ \\
Aggrecan 1 & 0.08 & & & & \\
Biglycan & 0.15 & $\mathbf{0 . 7 5}$ & $\mathbf{0 . 6 5}$ & $\mathbf{0 . 5 4}$ & $\mathbf{0 . 4 8}$ \\
Decorin & 0.84 & 1.26 & 0.61 & 0.58 & 0.55 \\
\hline
\end{tabular}

Table 3

Cell-matrix interactions - matrix metalloproteinases and inhibitors

\begin{tabular}{llllll}
\hline MMPs + TIMPs & \multicolumn{5}{l}{ Tendon sample } \\
\cline { 2 - 6 } & Normal & T1 & T2 & R1 & R2 \\
\hline MMP-1 & & & & & \\
MMP-2 & 0.13 & $\mathbf{0 . 6 7}$ & $\mathbf{0 . 5 3}$ & $\mathbf{0 . 4 9}$ & 0.09 \\
MMP-3 & 1.96 & & & & 0.24 \\
MMP-7 & & & & & \\
MMP-8 & & & & & \\
MMP-9 & & & & $\mathbf{0 . 0 3}$ & $\mathbf{0 . 0 4}$ \\
MMP-10 & & & & & \\
MMP-11 & & & & $\mathbf{0 . 0 3}$ & $\mathbf{0 . 0 4}$ \\
MMP-13 & 0.26 & $\mathbf{0 . 6 5}$ & $\mathbf{0 . 8 6}$ & $\mathbf{0 . 8 2}$ & 0.46 \\
MMP-14 & & & & & \\
MMP-15 & 0.07 & 0.05 & 0.04 & 0.04 & 0.01 \\
MMP-16 & & & & & 0.01 \\
MMP-17 & 0.07 & 0.08 & & 0.06 & 0.03 \\
MMP-19 & & & & & \\
MDC9 & 0.63 & 0.53 & 0.19 & 0.54 & 0.70 \\
TIMP-1 & 0.28 & 0.26 & 0.23 & 0.23 & 0.09 \\
TIMP-2 & 0.57 & 0.40 & 0.21 & 0.16 & 0.15 \\
TIMP-3 & & & & & \\
TIMP-4 & & 0.12 & 0.04 & 0.05 & 0.05 \\
$\alpha-2-m a c r o g l o b u l i n$ & & 0.38 & 0.09 & 0.25 & 0.30 \\
EMMPRIN & 1.21 & & & & \\
\hline
\end{tabular}

plotted (Fig. 3). Statistical analysis by the Wilcoxon signed rank sum test showed that the degenerate samples had significantly increased type-III and type-I collagen mRNA compared to 'normal' samples $(P=$ 0.05).

MMP-3 mRNA levels from the same pairs of samples were decreased in the isolates from degenerate tissue. The normalised levels of MMP-3 mRNA in the four normal cadaver samples varied considerably but were higher than those in the degenerate samples of the pairs (Fig. 4).

Results for real-time RT-PCR assay of MMP-2 and TIMP-3 mRNAs in RNA samples from the first six tendon pairs tested in duplicate are shown in Fig. 5. Unfortunately, there was insufficient RNA in any of the paired samples to allow for more replicates. MMP-1 mRNA levels in all samples were very low or undetectable.

\subsection{Protein extraction and immunoblotting for $M M P-3$}

The proteins isolated from the organic phase of the extraction reagent (with the exception of the 'normal' sample of tendon eight) gave similar patterns when separated by gel electrophoresis and stained with Coomassie Blue (Fig. 6a). Immunoblotting showed three of the four samples from individuals with no apparent tendon disorders had much higher levels of MMP-3 protein than those from patients with tendinosis (Fig. 6b). 

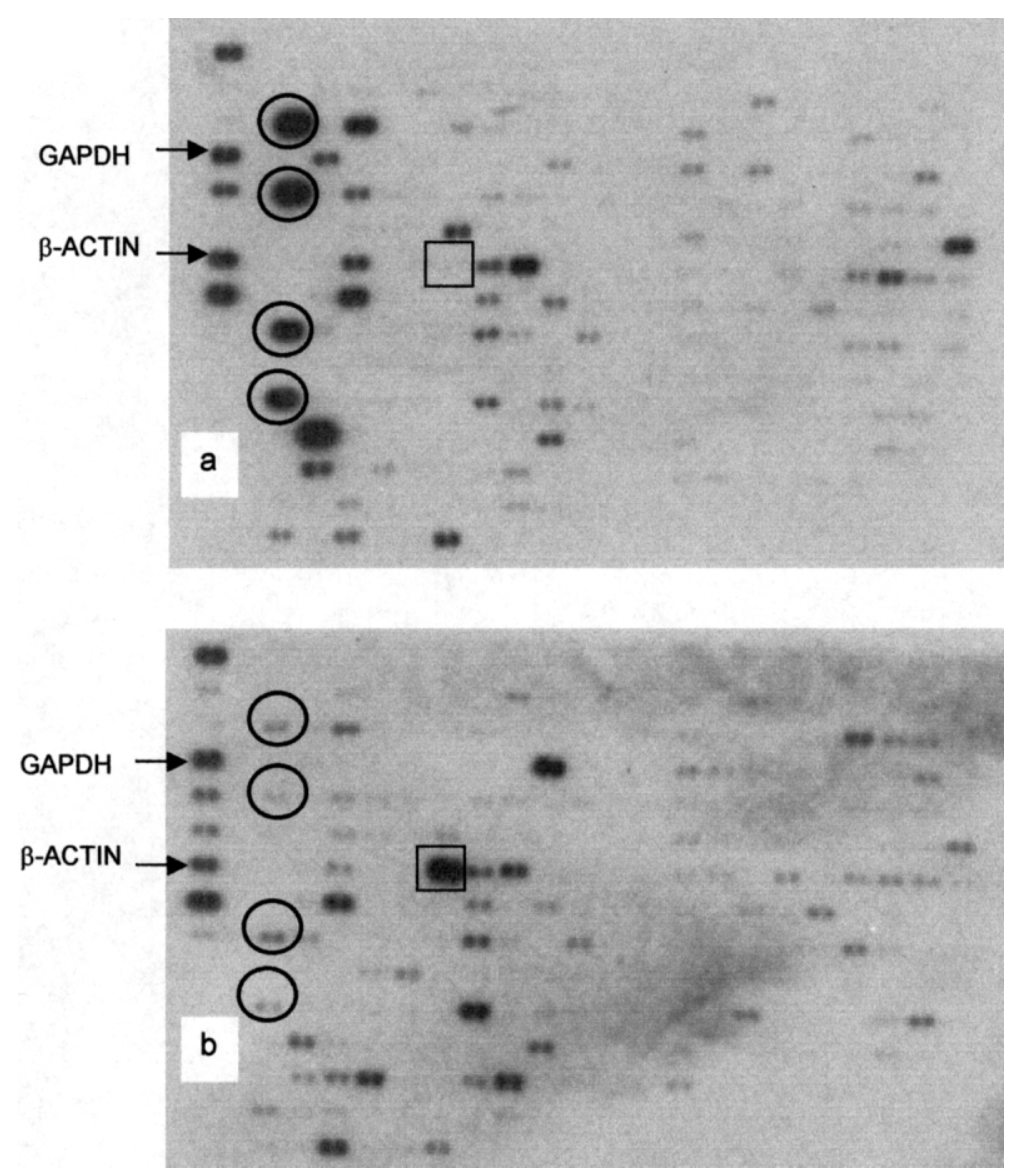

Fig. 1. Atlas ${ }^{\mathrm{TM}}$ cell-interaction arrays probed with ${ }^{32} \mathrm{P}$ labelled cDNA probes made with total RNA from human Achilles tendons; (a) degenerate tendon tissue (T1); (b) normal tendon tissue. Spots surrounded by circles are types-I, -III and -VI (alpha 1 and alpha 3) collagens, which are up-regulated in degenerate tendon tissue. Spots surrounded by a square are MMP-3, which is down-regulated in degenerate tendon tissue.

Tables 1-3 show expression of collagens, non-collagen matrix components, MMPs and inhibitors tested using cell-interaction arrays. Numbers are the ratios for gene-specific signal/ signal for GAPDH. Genes that showed increased expression ( $>$ two fold) in degenerate Achilles tendon tissue samples are shown in bold type while those that had increased expression in normal tissue ( $>$ two fold) are shaded.

\section{Discussion}

Surgery for chronically painful Achilles tendons is done at a late stage when non-operative treatment has failed. Tissue samples can be taken for immunohistochemical and biochemical analyses, that show the cumulative effects of cellular processes up to the time of biopsy. This analysis of gene expression in degenerate tissue from patients with Achilles tendinopathies and in the corresponding normal tissue from an age- and sex-matched cadaver has provided information about the regulation of tissue phenotype at the time that the samples were taken.

Marchuk et al. (1998) observed no degradation of rRNA or loss of integrity of mRNA isolated from rabbit ligament, tendon and cartilage up to $96 \mathrm{~h}$ post-mortem. Their findings indicate that normal human tendon tissue removed 2 days post-mortem was appropriate for this study. A pool of RNA extracted from a number of normal tendons would have been preferable to RNA from a single individual but the choice was dictated by the poor availability of cadaver tissue. The array results must be interpreted with caution since they do not take into account the variability of specific RNA levels in normal Achilles tendon. Moreover, the characteristics and variations of the hybridisation reactions make array data semiquantitative. Nonetheless, considered with both the quantitative RT-PCR results described here, and the results of earlier studies of tendinopathy, the findings do suggest a number of alterations in gene expression in tendinosis.

The ATLAS ${ }^{\mathrm{TM}}$ cell interaction arrays carry cDNAs 


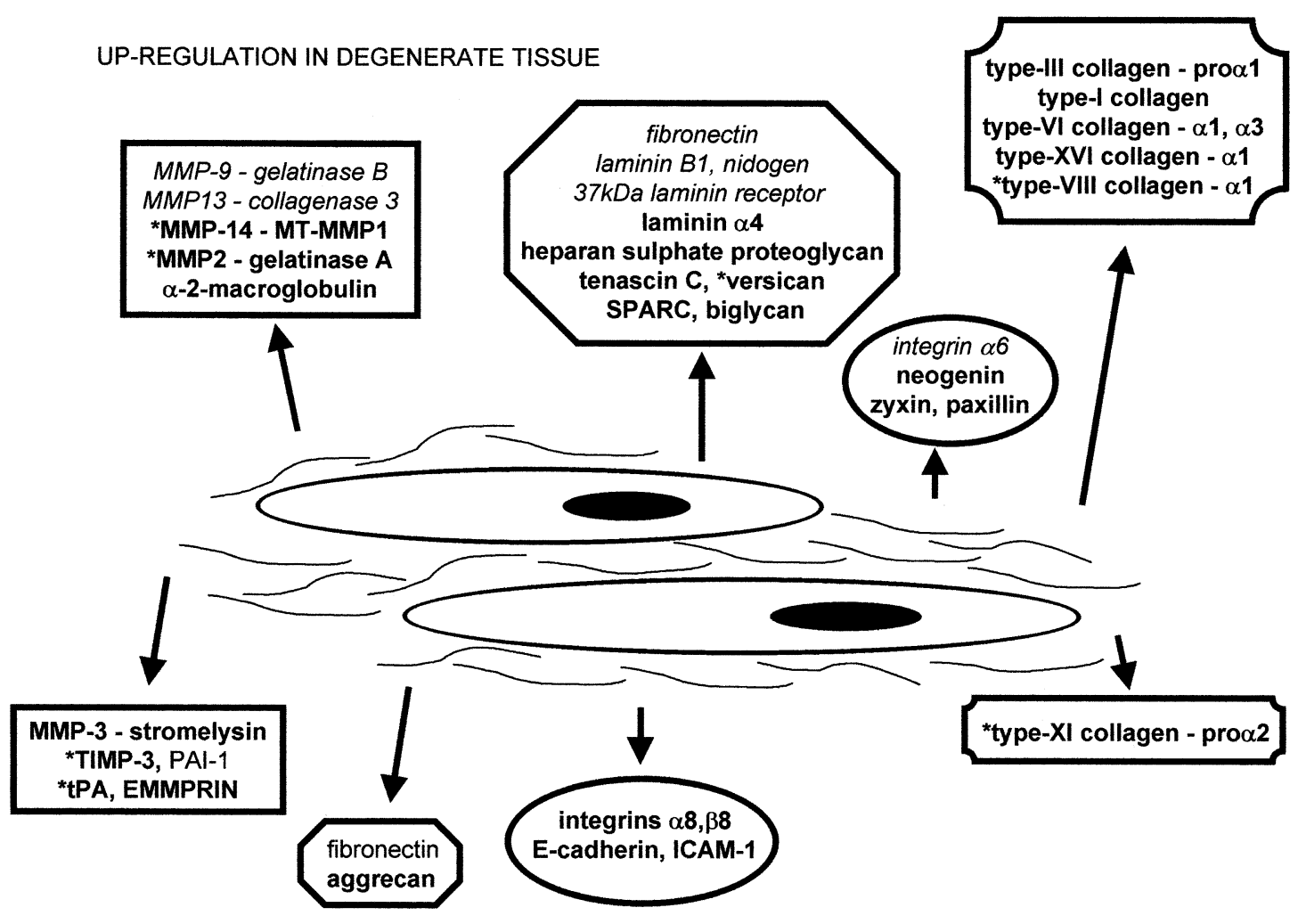

DOWN-REGULATION IN DEGENERATE TISSUE

Fig. 2. Differential expression of genes for ECM and cell-adhesion molecules in degenerate and normal human Achilles tendon tissue samples suggested by ATLAS ${ }^{\mathrm{TM}}$ cDNA arrays. Altered expression in all four is shown in bold type; in R1 and R2 only is shown in italic type; and in $\mathrm{T} 1$ and $\mathrm{T} 2$ only is shown in normal type.

for a number of the matrix metalloproteinases - a family of neutral enzymes that can degrade all the protein constituents of the ECM. Differences in MMP RNA expression between normal and degenerate Achilles tendon tissue samples were seen, with the biggest change being down-regulation of MMP-3 in the degenerate samples. RNAs from five pairs of tendon samples tested with five replicates by quantitative RT-PCR showed decreased MMP-3 RNAs in degenerate tissues. Thus, the differences seen on the gene arrays were not due to the different sources of normal and degenerate tissue and were localised within a tendon. The smaller differences between 'normal' and degenerate tissue samples from tendons that had lesions, and normal (post-mortem) and degenerate tissue samples, may reflect the difference between the two types of normal sample. Normalised levels of MMP-3 RNA in four normal cadaveric tendon tissue samples varied considerably but were higher than the levels in the degenerate samples. Immunoblotting of proteins extracted from the same tendon samples showed that three of the four normal tissue samples taken from individuals without apparent tendon disorder had much higher levels of MMP-3 protein than 'normal' or degenerate samples from patients with tendinosis.

MMP-3 is an MMP with a wide substrate range that includes most ECM components. It is also a potent activator of other MMPs. Thus, it has the potential to play a major role in regulation of tendon ECM degradation and tissue remodelling. Evidence for the importance of metalloproteases in maintaining human tendon health came from the tendinitic effects of marimastat, a broad-spectrum MMP inhibitor with weak anti-sheddase action, used as an anti-cancer agent. It is not clear which metalloproteases are involved since neither collagenase- nor gelatinasespecific inhibitors nor broad-spectrum MMP inhibitors with strong anti-sheddase action induced tendinitis in a rat model (Drummond et al., 1999). Sheddase activity is one of the functions of the adamalysins, cell surface metalloproteases with properties of both adhesion molecules and proteases (Killar et al., 1999). cDNAs for these enzymes are not included on the Atlas $^{\mathrm{TM}}$ cell-interaction arrays and their contribution to the degeneration of tendon tissue needs to be investigated using other tools.

The broad substrate range of MMP-3 includes pro- 


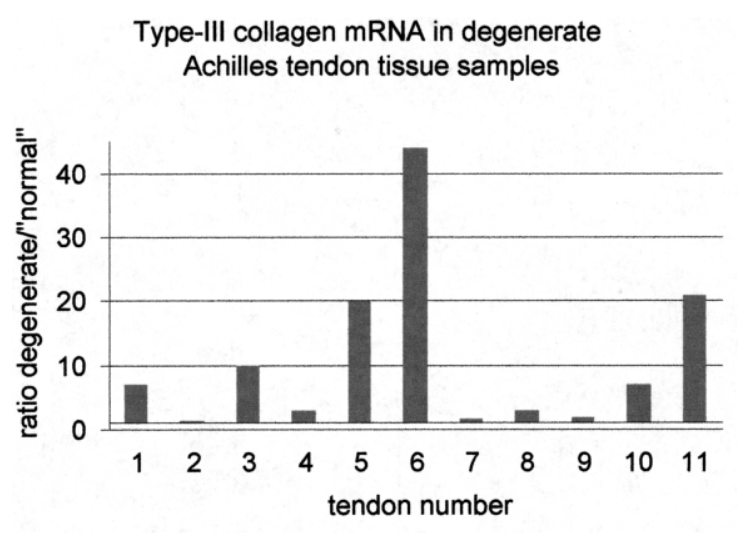

Type-I collagen mRNA in degenerate Achilles tendon tissue samples

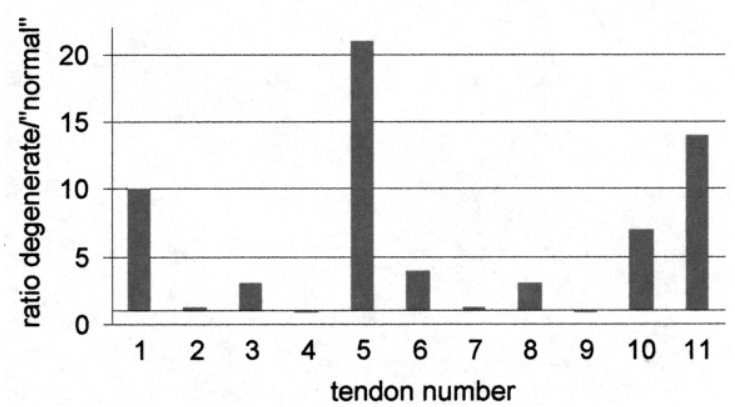

Fig. 3. RT-PCR assays for type-III and type-I collagen mRNAs in paired tissue samples from 11 patients with Achilles tendinosis. Both type-I and type-III collagen mRNAs were significantly increased in degenerate tendon tissue samples (Wilcoxon signed rank sum test: $P=0.05$ ).

teoglycans. Enhanced production of proteoglycans in degenerate tendon tissue was suggested by the observed increases in signals for perlecan, biglycan

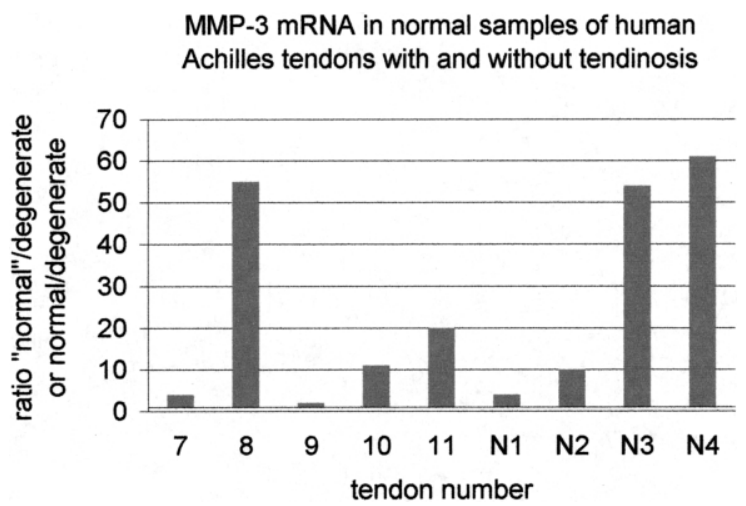

Fig. 4. Real-time RT-PCR assay for MMP-3 mRNA in human Achilles tendon tissue samples. Seven-11 - paired samples from patients with tendinosis. N1 to N4 - midsubstance tissue samples from normal cadaveric tendons. A single RNA preparation per sample was tested using five identical replicates. Ratios for normal samples were calculated using the average value of the five degenerate samples from the pairs.
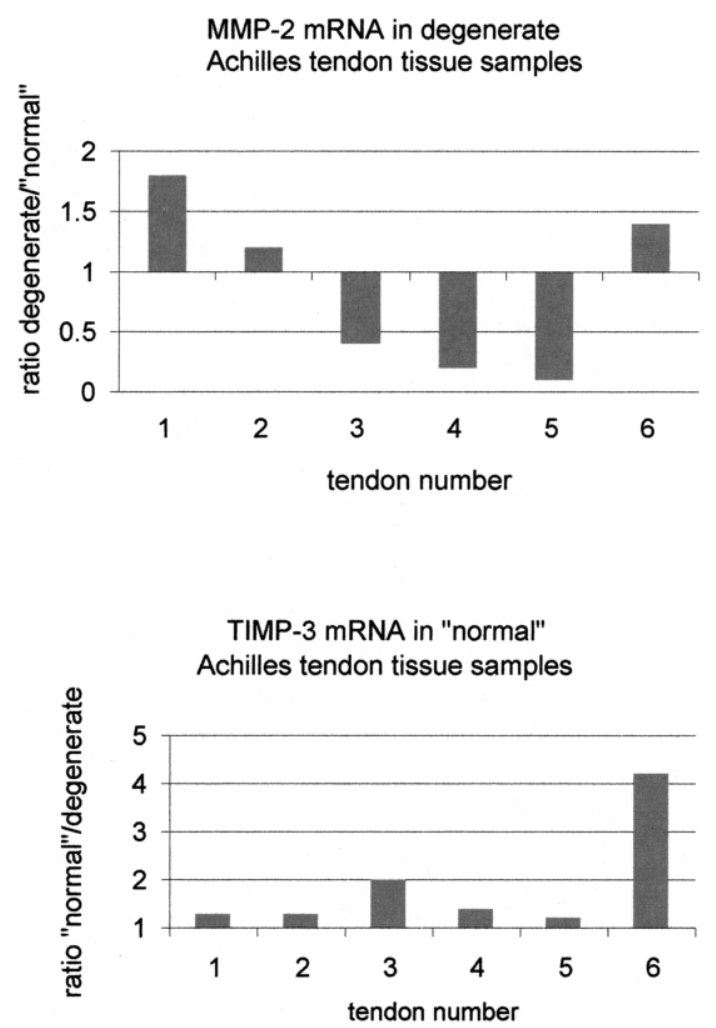

Fig. 5. Real-time RT-PCR assays for MMP-2 and TIMP-3 mRNAs in paired tissue samples from six patients with Achilles tendinosis. A single RNA preparation per sample was tested in duplicate.

and versican on the arrays. Increased proteoglycan synthesis with decreased MMP-3 activity would explain the greater Alcian blue glycosaminoglycan staining seen in degenerate Achilles tendon tissue sections. Increased heparan sulfate binding of growth factors may be responsible for the increased cellularity and angiogenesis seen in these tissues (Movin et al., 1997a).

MMP-1 RNA was not detected in the samples tested by gene array and the low levels of MMP-1 RNA in tendon tissue samples were confirmed by RT-PCR assay of paired samples. There was no evidence of increased levels of MMP-1 RNA in the degenerate tissues of the paired samples. Although MMP-1 and MMP-3 share homologous gene sequences and similar promoter elements, they do show differential expression in response to several stimuli, probably by recruiting different sets of transcription factors (Buttice et al., 1996).

Levels of MMP-2 (72 kDa gelatinase) and MMP-14 (MT1-MMP) RNAs were increased in three out of the four degenerate tendon tissue samples tested on the arrays. MMP-14 is important for the physiological activation of the inactive precursor form of MMP-2 (Strongin et al., 1995). However, RNAs from paired samples from six tendons tested in duplicate by RT- 
(a)

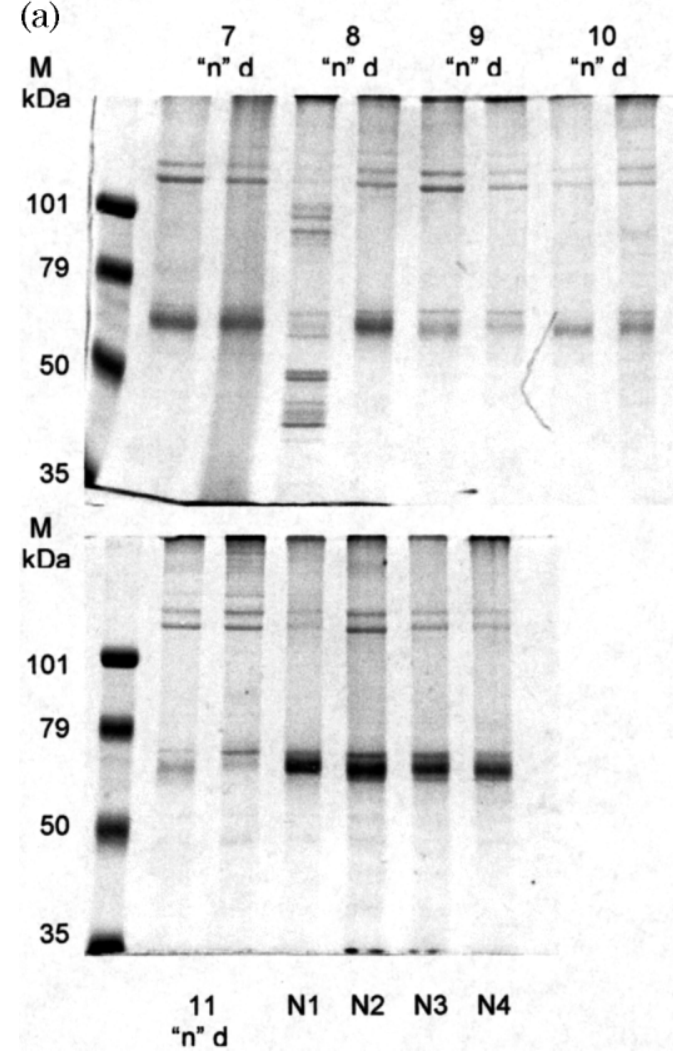

(b)
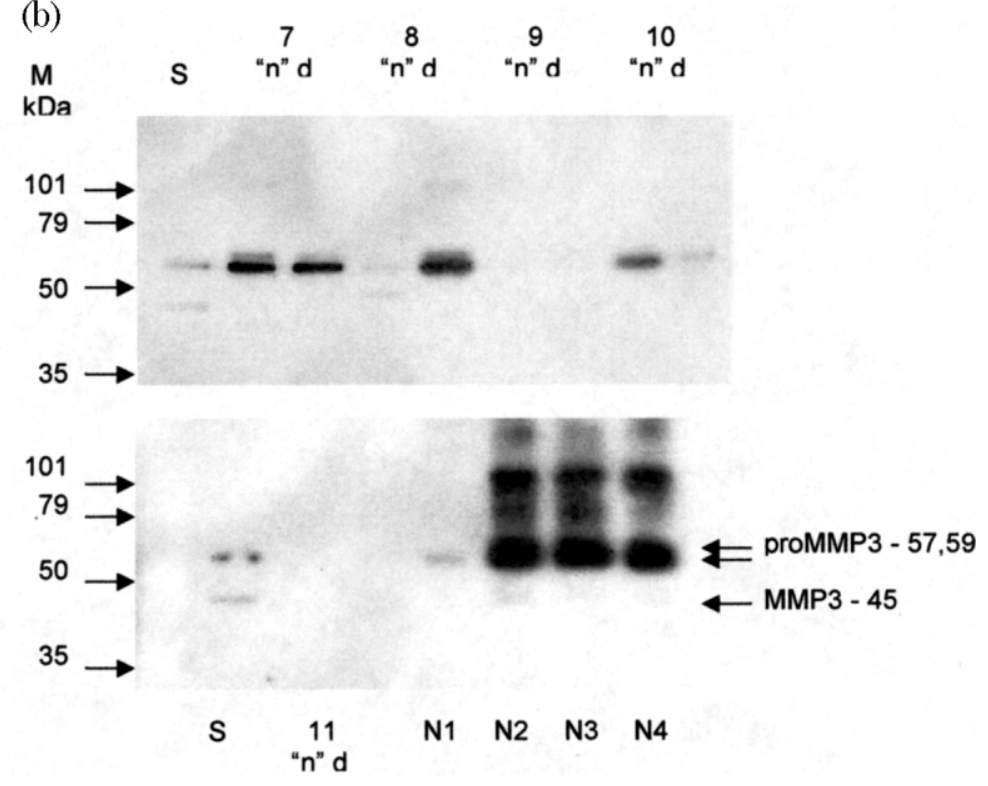

Fig. 6. Coomassie Blue-stained $10 \%$ polyacrylamide gel (A) and immunoblot (B) of MMP-3 using soluble protein extracted from human Achilles tendon tissue samples. M - molecular weight markers. S - purified MMP-3, 26 ng per track. Seven-11 - paired tendon tissue samples from patients with tendinosis; ' $n$ ' - macroscopically normal, $d$ - degenerate. N1-N4 - midsubstance tissue samples from normal cadaveric tendons. Soluble protein $(2 \mu \mathrm{g})$ isolated from tissue samples were loaded per track.

PCR showed no trend towards increased MMP-2 RNA in degenerate tissues.

Control over MMP activity involves inhibition of active enzymes and activation of zymogens as well as changes in gene expression. The balance of MMP and TIMP activities determines the extent of ECM turnover. We found no increases in TIMP RNA levels but did find decreases in TIMP-3 RNA in the degenerate tissue samples tested on arrays. RNAs from paired samples from six tendons tested in duplicate by RT-PCR showed a trend towards decreased TIMP-3 RNA in degenerate tissues, but there was insufficient material to confirm this with more replicates.

Differences in RNA levels for a number of collagen types were seen in the normal and degenerate tissue samples tested on the cDNA arrays. The greatest up-regulation in degenerate samples was in the genes for type-I and type-III collagens. RNAs from six pairs of tendon samples tested in duplicate by RT-PCR showed trends towards increased type-I and type-III collagen RNAs in degenerate tissues. Differences were significant when five replicates were used with RNAs from a further five pairs of samples. There was a greater increase in type-III collagen RNA than in
type-I collagen RNA in the degenerate tendon tissue samples tested. This finding is in keeping with earlier measurements of collagen proteins in tendinosis which showed high levels of type-III collagen protein in both degenerate equine tendon tissue (Birch et al., 1998) and spontaneously ruptured human Achilles tendon tissue (Coombs et al., 1980). Coombs and colleagues postulated that a high proportion of type-III collagen gives a tissue with reduced tensile strength and increased susceptibility to rupture.

We have previously suggested that tendon pathology may be linked to the development of fibrocartilage (Chard et al., 1993) but the low levels of type-II and type-IX collagen RNAs and of aggrecan RNA in the degenerate tendon tissue samples tested by array indicated an absence of chondrocyte-like cells. Rather, the array results suggest decrease in E-cadherin and increase in SPARC and tenascin C RNAs in degenerate samples, possibly resulting in loss of cell-cell contact (Sage and Bornstein, 1991). Unfortunately there was insufficient RNA from paired samples to measure RNAs for these molecules.

Previous studies have shown no evidence of inflammation in biopsies from degenerate tendon tissue 
(Movin et al., 1997a). The array results are in accordance with these findings since degenerate tendon tissue samples showed no increase in RNA for several markers of lymphocytes, monocytes and granulocytes (CD70, CD27, CD30 and CD33). Expression of CD4 RNA, expressed by $\mathrm{T}$ cells and macrophages, was down-regulated in degenerate compared to normal tendon tissue. RNAs for IL- 1 and TNF $\alpha$, important cytokine mediators of inflammation, were not detected on cDNA arrays.

Biopsy studies represent the end-stage of tendon disorders. It has been suggested that microtrauma caused by repetitive mechanical load leads to an early inflammatory phase followed by either healing or failure to heal with subsequent degenerative changes. Failure to heal has been attributed to decreased cell activity resulting from reduced vascularity and relative hypoxia at the site of the lesion (Alkeminders and Temple, 1998). This study demonstrates abundant cell activity in degenerate Achilles tendon tissue samples with increased expression of genes for type-I and type-III collagens, and probably of genes for proteoglycans and other non-collagen proteins. There is evidence that accumulation of proteoglycans results from increased proteoglycan synthesis coupled with reduced MMP-3 activity. Future studies using ultrasonography-guided percutaneous core biopsies taken at earlier stages of tendon disorders (Movin et al., 1997b) are needed to determine the stage at which the expression of genes becomes disregulated leading to tissue degeneration.

\section{Experimental procedures}

\subsection{Tendon specimens}

Four samples of degenerate mid-substance tendon tissue were obtained from patients undergoing surgery for Achilles tendon disorders. Two samples (T1 and T2) were from patients with chronic tendon pain (male, aged 51 and 53 years) and two (R1 and R2) from patients with spontaneously ruptured tendons (male, aged 50 and 52 years). The ruptures had happened 3-5 weeks prior to surgery. Normal control tissue was taken 2 days post-mortem from the corresponding regions of a human cadaver Achilles tendon (male, aged 56 years).

Paired tendon specimens were taken from 11 patients undergoing surgery for chronic tendon pain (male, average age 33 years, range $30-35$ ). One specimen of each pair came from the degenerate core region and the other from a nearby site that was macroscopically normal. The intervals between collection and freezing of tissue samples varied considerably; only the two samples in a given pair had identical treatment. Frozen samples were stored in liquid nitrogen or at $-80^{\circ} \mathrm{C}$ until used for RNA and protein extraction.

\subsection{RNA extraction and quantification}

RNA was extracted by a modification of the TriSpin method published by Reno et al. (1997). A rotorstator homogeniser was used to disrupt tendon tissue samples in $1 \mathrm{ml}$ of home-made monophasic reagent containing phenol, isoamyl alcohol, guanidinium isothiocyanate and beta-mercaptoethanol (Weber et al., 1998). The tissue samples were no more than $1 / 10 \mathrm{vol}$. of the reagent. After addition of $200 \mu \mathrm{l}$ of chloroform, and centrifugation, RNA was extracted from the aqueous phase using a commercially available RNA purification kit (SV Total RNA Isolation System - Promega), according to the manufacturer's instructions. RNA was eluted from the spin columns with $100 \mu l$ of RNase-free water and quantified fluorometrically using a RiboGreen kit (Molecular Probes) containing a fluorescent RNA dye. RNA needed for the preparation of labelled probes for cDNA expression array experiments was concentrated by alcohol precipitation after the addition of $1 / 10$ vol. of $2-\mathrm{M}$ sodium acetate. A sample of concentrated RNA was run on a denaturing gel to check that it was largely undegraded.

\section{3. $c D N A$ expression arrays}

cDNA probes were prepared from 1 to $2 \mu \mathrm{g}$ total RNA and hybridised to Atlas ${ }^{\mathrm{TM}}$ cell interaction arrays (CLONTECH) using the kit reagents according to the manufacturer's instructions. Three samples of normal tissue from the mid-portion of a human cadaver Achilles tendon and one sample of each of the patient Achilles tendons were used to probe the arrays. Labelled probes bound to the arrays were detected using BioMax MS film and intensifying screen (Kodak). The signals were quantified using Kodak 1D image analysis software. The signals for all the genes were then normalised to the signals for GAPDH and $\beta$-actin on the same array. The values for the four degenerate tissue samples were then compared to average values for the normal tissue. A gene was recorded as up-regulated or down-regulated if the difference was equal to or greater than two-fold. 


\subsection{RT-PCR primers and probes}

\subsubsection{Cloning and QC-RT-PCR}

GAPDH

Forward

Reverse

5'TGAAGGTCGGAGTCAACGGATTTG3'

5'GTTGGTGGTGCAGGAGGCATTGCT3'

Collagen type-I

Forward

5'CCCCCTCCCCAGCCACAAAGA3'

Reverse 5'TCTTGGTCGGTGGGTGACTCT3'

Collagen type-III

Forward

5'CCAAACTCTATCTGAA3'

Reverse 5'GGACTCATAGAATACA3'

MMP-1

Reverse

5'GAAAGGTGGACCAACAAT3'

5'ACTTCTCCCGAATCGTA3'

MMP-2

Forward

Reverse

5'CTACGACCGCGACAAGAAGTATG3'

3'TGCCAAGGTCAATGACAGGA3'

MMP-3

Forward

5'TCACAGACCTGACTCGGTT3'

Reverse

5'CACGCCTGAAGGAAGAGAGATG3'

TIMP-3

Forward

Reverse

5'CTTCTGCAACTCCGACATCGTG3'

5'TGCCGGATGCAGGCGTAGTGTTT3'
MMP-3

Forward 5'CCTTTGATGCTGTCAGCACTCT3'

Reverse 5'GCAATTCAGGTTCAAGCTTCCT3'

Probe 5'TTTGCGCCAAAAGTGCCTGTCTTTAAA

GAT3'

TIMP-3

Forward

Reverse

Probe

\author{
5'GCTGACAGGTCGCGTCTATGA3' \\ 5'GTAGCAGGACTTGATCTTGCAGTT3' \\ 5'AGCTGGTCCCACCTCTCCACGAAGTT3'
}

Forward

All primer pairs were chosen to include introns in the gene sequences. Primer Express ${ }^{\mathrm{TM}}$ software was used to design the primers and probes for use in the GeneAmp 5700 SDS. Probes were chosen to span intron-exon boundaries so that they were unable to hybridise to genomic DNA at the annealing temperatures of the PCRs.

\subsection{Preparation of $R N A$ standards and mimics for $Q C$ - RT-PCR}

A sample of total RNA from a specimen of degenerate Achilles tendon was reverse transcribed using random primers to give a pool of cDNA. This cDNA was used to prepare PCR products for cloning into the T-tailed vector pCR II-TOPO (Invitrogen) which has both SP6 and T7 RNA polymerase promotor sites. BamHI restriction sites were added to the forward primers so that the orientation of each insert could be determined by restriction of the plasmids with BamHI. PCR products for type-I and -III collagen, MMP-1 and GAPDH and the corresponding mimics were cloned. Forward primers extended at the $3^{\prime}$ end with nucleotides complementary to sequences approximately 60 bases downstream were used to create mimics with the same $5^{\prime}$ and $3^{\prime}$ sequences as the wild-type RNA, but with an internal deletion. This allowed gel separation of wild-type and mimic PCR products. Plasmids that contained inserts suitable for transcription with SP6 RNA polymerase were selected and cut with Not1. RNA transcripts were purified using Microspin S-300 columns (Pharmacia) after treatment with DNase1 to remove plasmid DNA. RNA was quantified using a RiboGreen kit.

\section{6. $Q C-R T-P C R$}

Forward

Reverse

Probe

5'TTTGATGTACCCTAGCTACACCTTCA3' 5'AAAGGTTAGCTTACTGTCACATGCTTT3' 5'CCAAGCCATATATGGACGTTCCCAAAA TCC3'

MMP-2

Forward

Reverse

5'TACGACCGCGACAAGAAGTATG3'

3'TTGTTGCCCAGGAAAGTGAAG3'

Probe
5'CCCTGAGACCGCCATGTCCACTGTT3'
Total RNA was used in a QC-RT-PCR method in which external standard curves were generated by co-amplification of increasing amounts of in-vitro transcribed wild-type RNA with a constant amount of competitor mimic RNA (Gerna et al., 1994). Access ${ }^{\mathrm{TM}}$ RT-PCR system reagents (Promega) were used for single tube RT-PCR, with the dNTP mix in the kit 
replaced by a PCR DIG labelling mix (Roche). PCR products were separated on $4 \% \mathrm{Hi}$-Sieve (Helena Biosciences) agarose gels, blotted onto positively charged nylon membrane, and fixed for $15 \mathrm{~min}$ at $120^{\circ} \mathrm{C}$. Labelled products were then detected using a sheep anti-DIG-alkaline phosphatase conjugate, CDP-Star ${ }^{\mathrm{TM}}$ (Roche), and ECL-film (Amersham). Individual bands from scanned films were quantified using the Kodak 1D image analysis program. The signal ratio wild-type/mimic was calculated for the standards and samples, and sample values were calculated from the standard curve. Values for specific mRNAs were normalised to GAPDH and expressed as normalised ratios.

\subsection{RT-PCR using GeneAmp 5700 SDS}

One-step RT-PCR reagents (Perkin-Elmer) were used in the GeneAmp 5700 SDS. A standard curve prepared by dilution of in-vitro transcribed RNA was included on each assay plate and the relative amounts of specific mRNA in the samples calculated from this curve. The first set (1-6) of tendon samples were tested in duplicate and average values used for calculations. The second set (7-11) of samples were tested using five identical replicates for each RNA sample, and the mean values and standard deviations were used to calculate the normalised ratios using the formula

$\mathrm{cv}=\sqrt{ } \mathrm{cv}_{1}^{2}+\mathrm{cv}_{2}^{2}$ where $\mathrm{cv}=$ S.D. $\div$ mean value

to calculate the standard deviations of the quotients. The means for each sample in the pairs were then compared using the approximate test for unequal variances based on the $t$ distribution (Armitage and Berry, 1994).

Since only one pair of samples could be taken from each patient, the results of the two methods of RTPCR were pooled to give a total of 11 pairs and the ratios degenerate/'normal' plotted. The results were analysed by the Wilcoxon signed rank sum test (Armitage and Berry, 1994).

\subsection{Extraction of soluble protein and immunoblotting for $M M P-3$}

Protein was isolated from the organic phase of the extraction reagent after removal of the aqueous phase containing RNA. After alcohol precipitation, the protein was dissolved in $1 \%$ SDS by heating for $30 \mathrm{~min}$ at $50^{\circ} \mathrm{C}$. The protein was quantified using the BCA protein assay reagent (Pierce) according to the manufacturer's instructions. PAGE was used to separate the polypeptides and gels were stained with Coomassie blue or blotted onto PVDF membrane. Purified hu- man MMP-3 (CC1035-Chemicon) was loaded onto the same gels. The membranes were stained using a mouse monoclonal antibody against MMP-3 (ZIF 7103 - TCS Biologicals), and reagents from the WesternBreeze ${ }^{\mathrm{TM}}$ chemiluminescent immunodetection system (Novex). The results were visualised on HyperfilmMP (Amersham) film.

\section{Acknowledgements}

This work was funded by grants from REMEDI, The Isaac Newton Trust, The Sybil Eastwood Memorial Trust and The Cambridge Arthritis Research Endeavour (CARE).

\section{References}

Alkeminders, L.C., Temple, J.D., 1998. Etiology, diagnosis and treatment of tendonitis: an analysis of the literature. Med. Sci. Sports Exerc. 1183-1190.

Armitage, P, Berry, G., 1994. Statistical Methods in Medical Research. Blackwell Science Ltd., pp. 111-113 (also pp. 450-451).

Astrom, M., 1998. Partial rupture in chronic Achilles tendinopathy. A retrospective analysis of 342 cases. Acta Orthop. Scand. 69, 404-407.

Astrom, M., Rausing, A., 1995. Chronic Achilles tendinopathy: a survey of surgical and histopathologic findings. Clin. Orthop. Relat. Res. 316, 151-164.

Birch, H.L., Bailey, A.J., Goodship, A.E., 1998. Macroscopic 'degeneration' of equine superficial digital flexor tendon is accompanied by a change in extracellular matrix composition. Equine Vet. J. 30, 534-539.

Buttice, G, Duterque-Coquillaud, M, Basuyaux, JP, Carrere, S, Kurkinen, M, Stehelin, D., 1996. Erg, an Ets-family member, differentially regulates human collagenase 1 (MMP1) and stromelysin 1 (MMP3) gene expression by physically interacting with the Fos/Jun complex. Oncogene 13, 2297-2306.

Chard, M.D., Cawston, T.E., Riley, G.P., Gresham, G.A., Hazleman, B.L., 1993. Rotator cuff degeneration and lateral epicondylitis - a comparative histological study. Ann. Rheum. Dis. $53,30-34$.

Coombs, R.R.H., Klenerman, H., Narcisi, P., Nichols, A., Pope, F.M., 1980. Collagen typing in Achilles tendon rupture. J. Bone Jt. Surg. 62B, 258.

Dahmen, G., 1964. Alterungs- und Degenerationsveranderungen des Bindegewebes in ihrer Bedeutung fur die Klink. Z. Rheumaforsch. 23, 393-405.

Drummond, A.H., Beckett, P., Brown, P.D., Bone, E.A., Davidson, A.H., Galloway, W.A., Gearing, A.J.H., Huxley, P., Laber, D., McCourt, M., Whittaker, M., Wood, L.M., Wright, A., 1999. Preclinical and clinical studies of MMP inhibitors in cancer. Ann. N.Y. Acad. Sci. 878, 228-235.

Gerna, G.F., Balfanti, F., Sarasini, A. et al., 1994. Effect of foscarnet induction treatment on quantitation of human cytomegalovirus (HCMV) DNA in peripheral blood polymorphonuclear leukocytes and aqueous humor of AIDS patients with HCMV retinitis. Antimicrob. Agents Chemother. 38, 38-44. 
Gibson, W., 1998. Are 'spontaneous' Achilles tendon ruptures truly spontaneous? Br. J. Sports Med. 32, 265-268.

Khan, K.M., Cook, J.L., Bonar, F., Harcourt, P., Astrom, M., 1999. Histopathology of common tendinopathies - Update and implications for clinical management. Sports Med. 27, 393-408.

Kannus, P., Jozsa, L., 1991. Histopathological changes preceding spontaneous rupture of a tendon. A controlled study of 891 patients. J. Bone Jt. Surg. (Am.) 73, 1507-1525.

Killar, L., White, J., Black, R., Peschon, J., 1999. Adamalysins. A family of metzincins including TNFa converting enzyme (TACE). Ann. New York Acad. Sci. 878.

Lukashev, M.E., Werb, Z., 1998. ECM signalling: orchestrating cell behaviour and misbehaviour. Trends Cell Biol. 8, 437-444.

Marchuk, L., Sciore, P., Reno, C., Frank, C.B., Hart, D.A., 1998. Post-mortem stability of total RNA isolated from rabbit ligament, tendon and cartilage. Biochim. Biophys. Acta Gen. Subj. 1379, 171-177.

Movin, T., Gad, A., Reinholt, F.P., Rolf, C., 1997a. Tendon pathology in long-standing achillodynia. Acta Orthop. Scand. 68, $170-175$.
Movin, T., Guntner, P., Gad, A., Rolf, C., 1997b. Ultrasonographyguided percutaneous core biopsy in Achilles tendon disorder. Scand. J. Med. Sci. Sports 7, 244-248.

Petruzzelli, L., Takami, M.D., Humes, H.D., 1999. Structure and function of cell adhesion molecules. Am. J. Med. 106, 467-476.

Reno, C, Marchuk, L., Sciore, P., Frank, C.B., Hart, DA., 1997. Rapid isolation of total RNA from small samples of hypocellular, dense connective tissues. Biotechniques 22, 1082-1086.

Sage, H., Bornstein, P., 1991. Extracellular proteins that modulate cell-matrix interactions: SPARC, tenascin and thrombospondin. J. Biol. Chem. 266, 14831-14834.

Strongin, A.Y., Collier, I., Bannikov, G., Marmer, B.L., Grant, G.A., Goldberg, G.I., 1995. Mechanism of cell surface activation of 72-kDa type IV collagenase. Isolation of the activated form of the membrane metalloprotease. J. Biol. Chem. 270, 5331-5338.

Weber, K., Bolander, M.E., Sarkar, G., 1998. PIG-B: A home-made monophasic cocktail for the extraction of RNA. Mol. Biotechnol. $6,73-77$. 